Acta Crystallographica Section D

Biological

Crystallography

ISSN 0907-4449

\author{
Navraj S. Pannu, ${ }^{a *}$ \\ Raimond B. G. Ravellib and \\ Jan Pieter Abrahams ${ }^{\mathrm{a}}$
}

aiophysical Structural Chemistry, Leiden Institute of Chemistry, Gorlaeus Laboratories, Leiden University, PO Box 9502, 2300 RA Leiden, The Netherlands, and ${ }^{\mathbf{b}} \mathrm{MCB}-\mathrm{EM}$, Leiden University Medical Center, PO Box 9600, 2300 RC Leiden, The Netherlands

Correspondence e-mail: raj@chem.leidenuniv.nl

\section{The Max-Inf2/Lorentz Center workshop on New algorithms in macromolecular crystallography and electron microscopy}

The resolution gap between macromolecular crystallography and electron microscopy continues to decrease. Recent advances in specimen preparation, instrumentation and computational power have allowed accurate structure determination of larger macromolecular complexes by crystallography and/or by electron microscopy on cryovitrified samples. New possibilities in structural biology have opened up and new challenges are faced to further reduce the resolution gap. A workshop at the Lorentz Center, Leiden, The Netherlands, which took place in May 2008, was organized to push further the limits of both complementary techniques through improved computational methods.

\section{Introduction}

On 12-18 May 2008, we held a workshop at the Lorentz Center, Leiden, The Netherlands for experts in computational methods development in macromolecular X-ray crystallography (MX) and electron microscopy (EM) to discuss algorithms of the future to maximally exploit the latest technology, improve both methods and combine the complementary information from both techniques optimally.

The general setup of the workshop involved morning lectures followed by informal 'open' discussions led by a group leader. Since the workshop brought together experts from two different fields, the workshop started with introductions to current approaches and challenges in both fields. Two afternoon sessions were reserved for talks selected from the submitted abstracts. The workshop was hosted by the Lorentz Center (http://www.lorentzcenter.nl/), where, in addition to a lecture room, meeting room and social corner, each participant had his/her own office with a desk, computer and white board.

This issue of Acta Cryst. Section D contains contributed manuscripts that were presented at the workshop.

\section{Workshop topics}

\subsection{Optimally combining multiple sources of information}

Macromolecular crystallography and electron microscopy can provide complementary information: combining this information optimally was a major focus of the workshop. Algorithms successfuly using EM maps as a starting model for $\mathrm{X}$-ray crystallographic molecular-replacement phasing were presented (i.e. Navaza, 2008) along with algorithms for placing models (Roseman, 2000; Fabiola \& Chapman, 2005; Siebert \& Navaza, 2009) or shapes (Heuser et al., 2009) in electrondensity maps. Validating the placement of atomic models was identified as an important area of research: current progress in 
developing statistics of docking atomic models in density maps was presented (Volkmann, 2009).

\subsection{Likelihood methods for MX and EM}

Likelihood methods are now widely accepted as the method of choice for obtaining the best parameters given the observations. Gerard Bricogne chaired the open discussion on likelihood methods in crystallography and presented his pioneering work on likelihood methods in crystallographic phasing (de La Fortelle \& Bricogne, 1997) and the work of Sigworth (1998) on a likelihood analysis of EM alignment. Advances on the successful application and further developments of likelihood methods for alignment and classification in electron microscopy (Scheres \& Carazo, 2009) were also presented.

\subsection{Cross-fertilization between $M X$ and EM}

The generality of likelihood functions allows them to be applicable for any parameterization. Ma and coworkers (Ni et al., 2009) have shown that parameterization in normal modes is beneficial, particularly at low resolution. Another important subject of this workshop was to reduce the resolution gap between X-ray crystallography and electron microscopy. Essential for this approach is identifying a weak solution amongst noisy peaks: cluster analysis is shown to be a useful discriminator in these circumstances (Buhler et al., 2009). Access to a unique database of structural domains can open up new possibilities for docking models in low-resolution maps or for application in molecular replacement (Long et al., 2008). A novel algorithm was presented to index, process and combine X-ray or electron diffraction data that are obtained from many small, weakly diffracting and randomly oriented crystals (Jiang et al., 2009). All computational methods in MX and EM rely on the accurate acquisition of data in digital form. A computational toolbox was presented (Vulovic et al., 2009) to evaluate the performance of CCD devices used in EM and a comparison with detectors for MX was made.

\subsection{Solutions for demanding algorithms}

More sophisticated algorithms often require greater computational power. Diederichs (2000) presented the use of programming paradigms for exploiting parallel hardware to speed up crystallographic applications. Schmeisser et al. (2009) chaired an open discussion illustrating the use of CUDA for scientific programming on GPUs. A demonstration was given with a program used for single-particle electron microscopy.

We thank Gerda Filippo and Martje Kruk for their efforts in ensuring a well organized workshop. The success of the conference was due to the speakers: Marc Schiltz, Abraham Koster, Thomas Schneider, Marc Storms, Jianpeng Ma, Dilyana Georgieva, Alexandre Urzhumtsev, Ozan Öktem, Garib Murshudov, Sjors Scheres, Kevin Cowtan, Gerard Bricogne, Achilleas Frangakis, Kay Diederichs, Holger Stark, Vince Fernando, Alan Roseman, Martin Schmeisser, Burkhard Heisen, Dominik Moser, Wijnand Mooij, Jorge Navaza, Michael Chapman, Alexei Vagin, Xavier Siebert, Niels Volkmann, Roeland Boer, Igor Orlov, Victor Lamzin and Marin van Heel. This meeting was made possible by the generous financial support from Max-Inf2, the Lorentz Center, Cyttron, FEI and Bruker.

\section{References}

Buhler, A., Urzhumtseva, L., Lunin, V. Y. \& Urzhumtsev, A. (2009). Acta Cryst. D65, 644-650.

Diederichs, K. (2000). J. Appl. Cryst. 33, 1154-1161.

Fabiola, F. \& Chapman, M. S. (2005). Structure 13, 389-400.

Heuser, P., Langer, G. G. \& Lamzin, V. S. (2009). Acta Cryst. D65, 690-696.

Jiang, L., Georgieva, D., Zandbergen, H. W. \& Abrahams, J. P. (2009). Acta Cryst. D65, 625-632.

La Fortelle, E. de \& Bricogne, G. (1997). Methods Enzymol. 276, 472494.

Long, F., Vagin, A. A., Young, P. \& Murshudov, G. N. (2008). Acta Cryst. D64, 125-132.

Navaza, J. (2008). Acta Cryst. D64, 70-75.

Ni, F., Poon, B., Wang, Q. \& Ma, J. (2009). Acta Cryst. D65, 633-643.

Roseman, A. M. (2000). Acta Cryst. D56, 1332-1340.

Scheres, S. H. W. \& Carazo, J.-M. (2009). Acta Cryst. D65, 672-678.

Schmeisser, M., Heisen, B. C., Luettich, M., Busche, B., Hauer, F., Koske, T., Knauber, K.-H. \& Stark, H. (2009). Acta Cryst. D65, 659671.

Siebert, X. \& Navaza, J. (2009). Acta Cryst. D65, 651-658.

Sigworth, F. J. (1998). J. Struct. Biol. 122, 328-339.

Volkmann, N. (2009). Acta Cryst. D65, 679-689.

Vulovic, M., Reiger, B., van Vliet, L., Koster, A. \& Ravelli, R. (2009). Submitted. 